\title{
Switching among pulse-generation regimes in passively mode-locked fibre laser by adaptive filtering
}

\author{
Junsong Peng, Sonia Boscolo \\ Aston Institute of Photonic Technologies, School of Engineering and Applied Science, Aston \\ University, Birmingham B4 7ET, United Kingdom
}

\begin{abstract}
We show both numerically and experimentally that dispersion management can be realized by manipulating the dispersion of a filter in a passively mode-locked fibre laser. A programmable filter the dispersion of which can be software configured is employed in the laser. Solitons, stretched-pulses, and dissipative solitons can be targeted reliably by controlling the filter transmission function only, while the length of fibres is fixed in the laser. This technique shows remarkable advantages in controlling operation regimes in ultrafast fibre lasers, in contrast to the traditional technique in which dispersion management is achieved by optimizing the relative length of fibres with opposite-sign dispersion. Our versatile ultrafast fibre laser will be attractive for applications requiring different pulse profiles such as in optical signal processing and optical communications.
\end{abstract}

Keywords: Passively mode-locked fibre lasers, pulse shaping, frequency filtering, nonlinear fibre optics

\section{INTRODUCTION}

Passively mode-locked fibre lasers have been intensively investigated due to their wide applications $[1,2]$. The interplay among the effects of gain/loss, dispersion and nonlinearity can also be used to shape the pulses and manipulate the light dynamics and, hence, lead to different regimes of mode locking. When the group-velocity dispersion (GVD) of the laser is anomalous, the balance between nonlinearity and dispersion results in soliton formation in a laser $[3,4]$. The energy achievable in such laser systems is limited by the soliton area theorem. In a laser with segments of nearly equal magnitudes of GVD but with opposite signs, a pulse will stretch and compress experiencing lower nonlinearity. This stretched-pulse or dispersion-managed (DM) soliton [5-7] operation exists for net anomalous or small normal GVD and allows femtoseconds pulses with up to nanojoule energies. Recent work [8] has shown that much higher pulse energies can be reached in fibre lasers that operate at all-normal or strong net normal dispersion regime. In the normal-dispersion regime, dissipation is required and plays a key role in the pulse shaping, and similaritons $[9,10]$ or dissipative solitons (DSs)[11] can be found in this regime, depending on the laser parameter especially the gain[12]. The similariton features parabolic pulses that evolve self-similarly in a long segment of passive fibre [9]. Parabolic amplifier similaritons have also been stabilized in a fibre oscillator [10,13-15]. The amplifier similariton laser relies on a local nonlinear attraction to stabilize the pulse in the cavity, while spectral filtering plays an important supporting role by undoing the large spectral broadening after the gain segment. It is worth to mention that the soliton-similariton laser reported in [13, 14] is the only example of mode-locked laser so far that has two types of nonlinear waves propagating within the cavity. DSs features rectangular pulse spectrum. To date, the best energy performance from SMF-fibre lasers has been achieved with this mode-locking mechanism.

Techniques for generating specialized waveforms [16] have become increasingly important in many scientific areas, including, amongst others, ultrahigh-speed optical communications, and optical signal processing. Versatile ultrafast laser sources, which can selectively emit different types of pulses, are highly desirable in this context. The key to access different pulse regimes in passively mode-locked fibre laser is in-cavity dispersion management [17-20]. Commonly employed methods to achieve in-cavity dispersion tuning include grating pairs, or simply physically changing the length of the fibre in the cavity [17-20]. Furthermore, the possibility to achieve both parabolic self-similar and triangular pulse shaping in a mode-locked fibre laser via adjustment of the net normal dispersion and integrated gain of the cavity was reported in [21]. In [22], careful control of the gain/loss parameters of a net-normal dispersion laser cavity provided the

\footnotetext{
Laser Sources and Applications III, edited by Jacob I. Mackenzie, Helena Jelĺnková,

Takunori Taira, Marwan Abdou Ahmed, Proceedings of SPIE Vol. 9893, 98930I

(C) 2016 SPIE · CCC code: 0277-786X/16/\$18 · doi: 10.1117/12.2225141
} 
means of achieving switching among Gaussian pulse, DS and similariton pulse solutions in the cavity. All these techniques however, require manual tuning of some physical parameters of the cavity.

Spectral pulse shaping [23] employed in a mode-locked fibre laser has emerged as a method to achieve a potentially high degree of control over the dynamics and the output of the laser [24-28] purely through software control. This method was demonstrated to allow for tunability of the laser wavelength and laser operation at high repetition rates [28]. In $[29,30]$, the phase-filtering ability of an in-cavity pulse shaper was shown to enable precise control of the cavity dispersion of the laser as well as to change the output pulse train from bright to dark pulses. In [27], it was numerically shown that a passively mode-locked fibre laser can operate in different pulse-shaping regimes, including bright and dark parabolic, flat-top, triangular, and saw-tooth waveform generations, depending on the amplitude profile of an in-cavity spectral filter. Further, an application of this technique using a flat-top spectral filter was numerically demonstrated to achieve the direct generation of high-quality sinc-shaped optical Nyquist pulses with widely tunable bandwidth from a passively mode-locked fibre laser [28].

In this work, we demonstrate that different pulse regimes including soliton, DM soliton, and DS mode-locking regimes can be switched and reliably targeted by programming the dispersion and bandwidth on an in-cavity programmable filter. The generation and in-cavity evolution of the different regimes are further confirmed by a numerical analysis. Each of these mode-locking regimes obviously takes on major practical importance. To our knowledge, this is the first time that these distinctly different pulse solutions are obtained in a single laser system without applying any physical changes in the layout of the laser cavity.

\section{RESULTS AND DISCUSSION}

\subsection{Setup and experimental results}

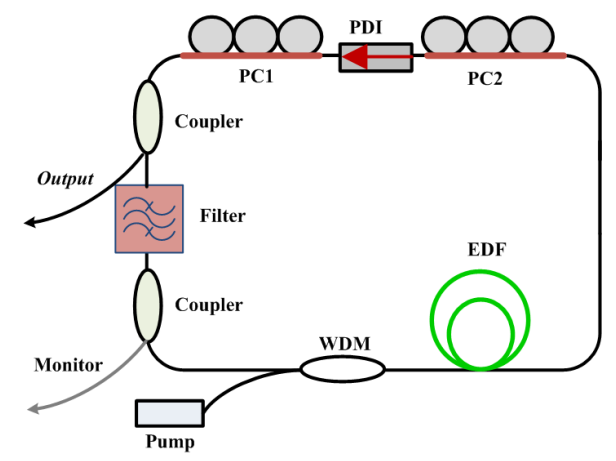

Figure 1. Schematic of filter-based dispersion-managed passively mode-locked Er-doped fibre laser. WDM: wavelengthdivision multiplexer; EDF: erbium-doped fibre; PC: polarization controller; PDI: polarization-dependent isolator.

The laser setup in the ring cavity configuration is shown in Fig. 1. A 1.2-m long segment of EDF with nominal absorption coefficient of $\sim 52 \mathrm{~dB} / \mathrm{m}$ at $976 \mathrm{~nm}$ and normal GVD $\beta_{2}=65.05 \mathrm{fs}^{2} / \mathrm{mm}$ at $1550 \mathrm{~nm}$ was used as the gain medium. This fibre was pumped through a 980/1550 wavelength-division multiplexer (WDM) by a 976-nm laser diode. An in-fibre polarization-dependent isolator (PDI) sandwiched with two polarization controllers (PCs), converted nonlinear polarization rotation (NPR) to amplitude modulation, initiating and stabilizing mode-locked operation [29, 30], and it also ensured single direction oscillation. Additional anomalous-dispersion fibre associated with the various components of the laser cavity amounted to a length of $\sim 8 \mathrm{~m}$. A programmable phase and amplitude filter was used to realize different regimes of pulse generation in the cavity. Under mode-locking conditions, the laser operated at a repetition period of $89 \mathrm{~ns}$, which remained constant throughout the experiments described below (since there was no change in the optical length of the cavity). The net dispersion of the cavity was adaptively managed by changing the curvature of the parabolic spectral phase profile of the filter (Section 2.2). As pulse formation at net anomalous or slightly normal GVD does not depend on spectral filtering, for such dispersion regimes we employed the default rectangular spectral profile of the filter. The filter's bandwidth was set to $9 \mathrm{THz}(\sim 73 \mathrm{~nm})$. On the other hand, for operation of the laser in the strong net-normal dispersion regime, the filter was programmed to have a narrow-bandwidth Gaussian spectral profile, as detailed below. A filter's center wavelength of $1555 \mathrm{~nm}$ was used for all dispersion regimes in use. Two 90:10 fibre couplers were employed to tap 10\% of laser power out of the cavity, where one was for laser 
outputs after the NPR components; the other one was located after the programmable filter for investigating the pulse dynamics inside the laser cavity. The output port was connected by a 3-m long segment of anomalous-dispersion SMF $\left(\beta_{2}=-22.8 \mathrm{fs}^{2} / \mathrm{mm}\right.$ at $\left.1550 \mathrm{~nm}\right)$ to an optical spectrum analyzer, an auto-correlator from Femtochrome and a fast photodetector $(1 \mathrm{GHz})$ connected to an oscilloscope $(2 \mathrm{GHz})$ to characterize the pulses.

When there was no dispersion applied on the programmable filter, the cavity had an anomalous net dispersion. By properly adjusting the two PCs in the system, stable mode-locked pulses could be obtained. A typical pulse train can be observed on the oscilloscope. In this example, the average pulse power after the SMF of the laser output port was 0.15 $\mathrm{mW}$. The corresponding optical spectrum profile at the laser output, was centered at $\sim 1560 \mathrm{~nm}$ and with a spectral bandwidth at full-width at half-maximum (FWHM) of $4.7 \mathrm{~nm}$. The presence of Kelly sidebands [31]in the spectrum indicates the fundamental soliton shape of the output pulse. The net dispersion of the cavity, calculated from the sidebands, is $-0.399 \mathrm{ps}^{2}$ (at $1560 \mathrm{~nm}$ ). The autocorrelation trace (not shown here) corresponds to FWHM pulse duration of $0.8 \mathrm{ps}$ when a hyperbolic secant fit is assumed. This gives a time-bandwidth product (TBP) of 0.46 , indicating that the pulse is slightly chirped. The soliton-like single-pulse generation regime was observed for a pump power of 20 to 26 $\mathrm{mW}$, and bore an average output power of 0.12 to $0.21 \mathrm{~mW}$ corresponding to a pulse energy 10.7 to $18.7 \mathrm{pJ}$. Multiple solitons were generated in the system for pump powers above $26 \mathrm{~mW}$. When some normal dispersion was applied on the filter while keeping the net cavity dispersion anomalous, the spectral width of the pulses increased, which is in agreement with soliton-type pulse behavior [2]. As an example, Fig. 2 shows the soliton spectrums under different net cavity dispersion.

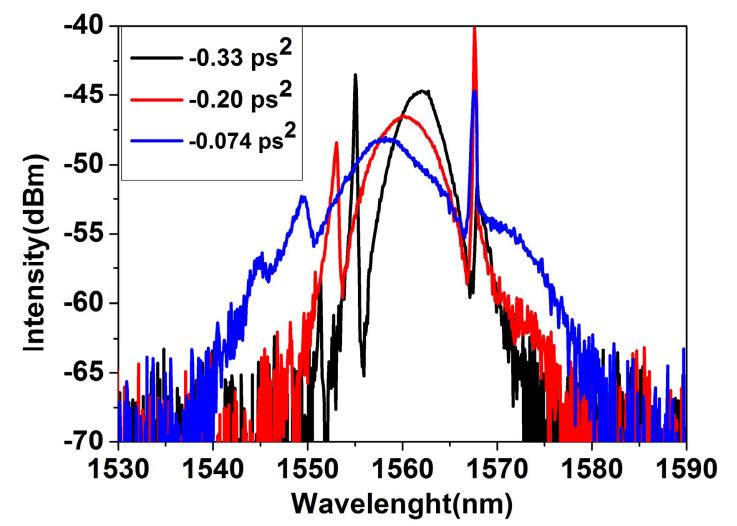

Figure 2. Typical soliton spectra when the net cavity dispersion is tuned with the filter.

Next, we applied a dispersion of $0.385 \mathrm{ps}^{2}$ (calculated at $1555 \mathrm{~nm}$ ) on the filter to shift the net cavity GVD close to zero value. In this case, the laser generated pulses with Kelly sideband-free, wide spectrum as seen in Fig. 3 (left), which is a signature of the DM soliton operation regime. An even wider output pulse spectrum was obtained by establishing the net GVD to be slightly normal [2] ( $\left.0.014 \mathrm{ps}^{2}\right)$. Single-pulse DM soliton mode locking existed under a pump power of 28.5 to $42 \mathrm{~mW}$, and bore an average output power of 0.24 to $0.41 \mathrm{~mW}$, yielding a pulse energy of 21 to $36 \mathrm{pJ}$, which is significantly higher than that of soliton-like mode locking. Further increase in the pump power resulted in multiple pulse generation. The autocorrelation trace of the pulse with $0.20 \mathrm{~mW}$ average power after the SMF of the laser output port is shown in Fig. 3 (right), and indicates a FWHM pulse duration of $2.78 \mathrm{ps}$ when a Gaussian fit is assumed.

It is well known that DS occur in all-normal [11, 32] or strong net-normal dispersion cavities [33, 34]. In our laser design, strong net-normal dispersion can be achieved by configuring the dispersion of the in-cavity filter; meanwhile, the filter's bandwidth also needs to be properly controlled as DS require additional amplitude modulation besides saturable absorber action [11,35]. Further, a Gaussian spectral profile was programmed on the filter, as the default rectangular profile is similar to the shape of the DS spectrum. Fig. 4 give the characterizations of the laser output obtained for a filter's bandwidth at FWHM of $0.8 \mathrm{THz}$ and filter's dispersion of $0.8986 \mathrm{ps}^{2}$ yielding a net GVD of $0.502 \mathrm{ps}^{2}$ in the cavity. No stable mode-locked pulses were found when the bandwidth of the filter was larger than $0.8 \mathrm{THz}$, consistently with the discussion above. The output pulse spectrum is steep at the edges and has a dip in the top (left), typical characteristics of DS lasing [8]. The spectral width at FWHM is $9.7 \mathrm{~nm}$. Incidentally, we also note that the spectrum is centered at the central wavelength of the filter, which confirms the ability of an in-cavity programmable filter to tune the 
laser wavelength [24]. The average output power for the DS mode-locking regime was 0.45 to $0.69 \mathrm{~mW}$ under the pump power 56.5 to $72 \mathrm{~mW}$, giving a pulse energy 39 to $61 \mathrm{pJ}$. The autocorrelation trace of the pulse with 0.48 - $\mathrm{mW}$ average power after the SMF of the laser output port reveals that the FWHM pulse duration is 3.5 ps when a Gaussian fit is assumed. This gives a TBP of 4.18, indicating that the pulse is highly chirped inside the cavity[8]. Laser generated DS were found to exist in the net cavity dispersion range 0.247 to $0.502 \mathrm{ps}^{2}$, and their spectral width decreased when the incavity GVD was increased, as it was expected.

Here we summarized results for the scaling of the laser output power with pump power for the different pulsegeneration regimes. The DS regime features the highest pumping threshold for mode locking and, correspondingly, the highest output pulse energy, in accordance with previous findings. The efficiency of power transfer from the pump to the laser signal is also the highest for the DS regime, owing to the large chirp of the pulses in the cavity.
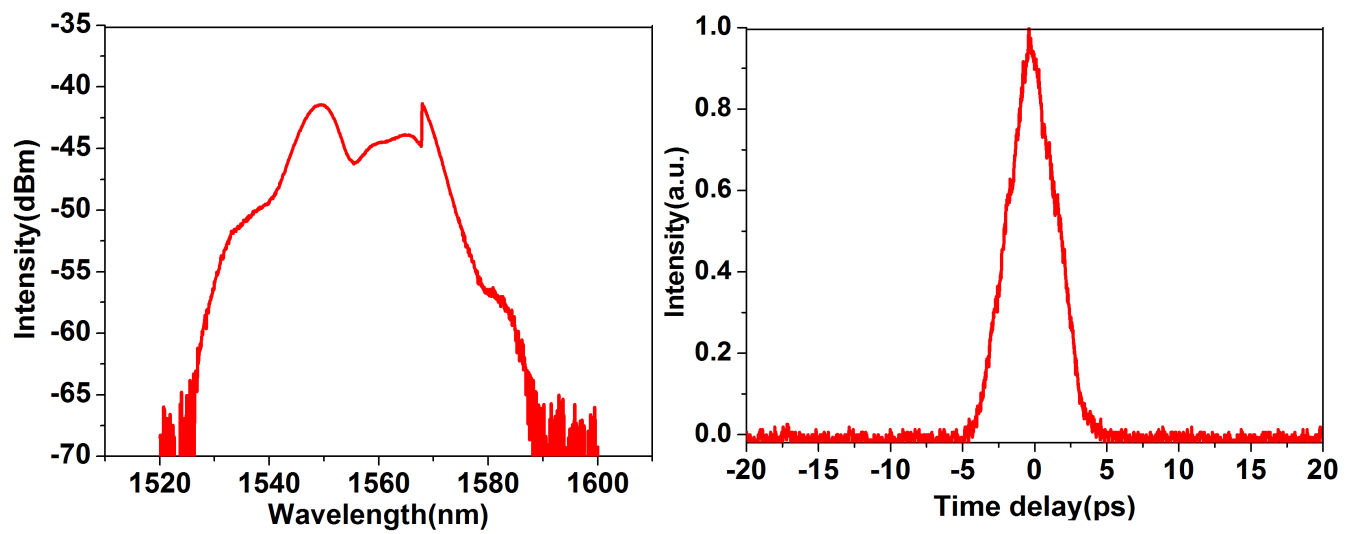

Figure 3. DM soliton spectrum (left) and its autocorrelation trace (right).
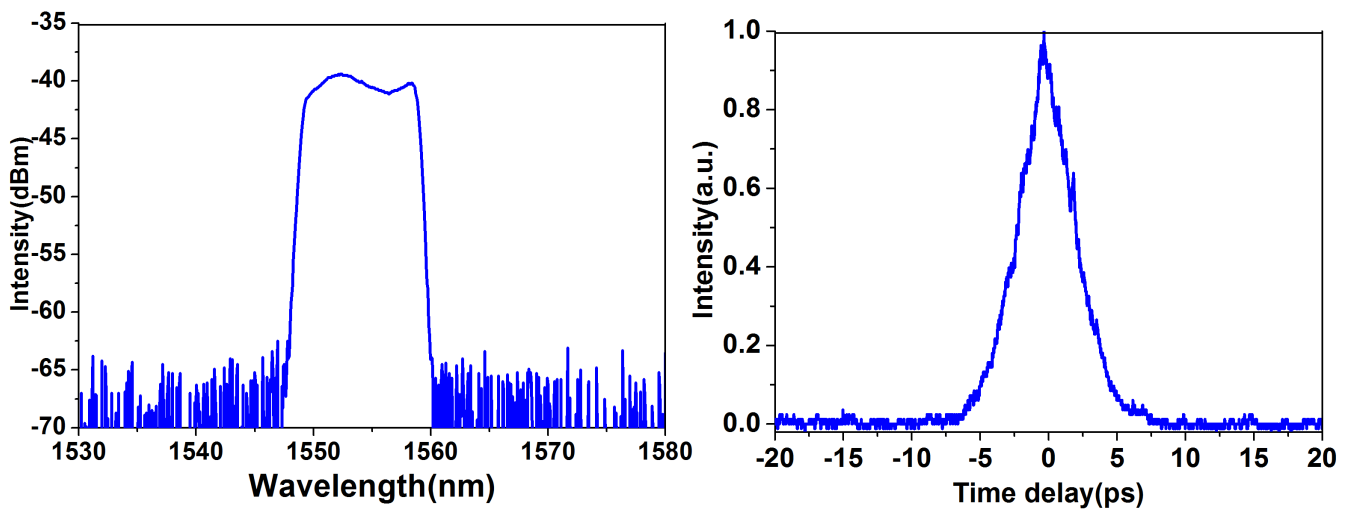

Figure 4. DS spectrum (left) and its autocorrelation trace (right).

\subsection{Numerical simulations}

To gain insight into the pulse dynamics inside this fibre laser cavity, we performed numerical simulations of the laser. The laser configuration used in the simulations was the same as the experimental setup. Pulse propagation within the fibre sections was modelled with a standard modified nonlinear Schrödinger equation for the slowly varying pulse envelope [40]:

$$
\frac{\partial \psi}{\partial z}=-\frac{i}{2}\left(\beta_{2}+i \frac{g}{\Omega^{2}}\right) \frac{\partial^{2} \psi}{\partial t^{2}}+i \gamma|\psi|^{2} \psi+\frac{1}{2} g \psi
$$

Here $\beta_{2}$ is the GVD parameter and $\gamma$ is the coefficient of cubic nonlinearity for the fibre section. The dissipative terms 
represent linear gain as well as a parabolic approximation to the gain profile with the bandwidth $\Omega$. The gain is described by $g=g_{0} /\left(1+W / W_{0}\right)$, where $g_{0}$ is the small-signal gain (corresponding to $30 \mathrm{~dB} / \mathrm{m}$ in power), which is non-zero only for the gain fibre, $W=\int|\psi|^{2} d t$ is the pulse energy, and $W_{0}$ is the gain saturation energy determined by the pump power. To initiate and sustain mode locking of the fibre laser, the NPR technique was used in our experiment. Here the mode-locking regime for the sake of clarity was modelled by a simple transfer function [36]: $T=1-q_{0}-$ $q_{m} /\left[1+P(t) / P_{0}\right]$, where $q_{0}$ is the unsaturated loss, $q_{m}$ is the saturable loss (modulation depth), $P(t)$ is the instantaneous pulse power, and $P_{0}$ is the saturation power. The filter was modelled by the spectral response [27]: $H(f)=R(f) \exp \left[i \beta_{2, \text { acc }}(2 \pi f)^{2} / 2\right]$, where the spectral phase added a specific amount of GVD $\beta_{2, \text { acc }}$ to the cavity to control the net cavity dispersion. We used a rectangular spectral profile $R(f)=\operatorname{rect}(f / B)$ with the bandwidth $B=10$ $\mathrm{THz}$ to operate the laser at anomalous or slightly normal dispersion, and a Gaussian spectral profile $R(f)=$ $\exp \left[-f^{2} /\left(2 B^{2}\right)\right]$ with a FWHM bandwidth of $0.8 \mathrm{THz}$ for the strong net-normal dispersion regime, in accordance with the experiment. To account for additional length of anomalous-dispersion fibre in the cavity, we included two segments of SMF after the saturable absorber (SA) element and the filter, respectively. Linear losses of $10 \%$ were imposed after the SMF segment following the SA and after the filter, which summarized intrinsic losses and output coupling. The laser output was monitored behind the coupler at the exit of the SMF segment following the SA, after further propagation in a 3-m-long segment of SMF as in the case of the experiment. The parameters used in the numerical simulations were similar to their nominal or estimated experimental values. We would like to point out that we did not aim here at a comprehensive comparison of numerical modeling and experiments and intentionally considered a simplified description of some key effects. Instead, we used this simple model to highlight the main features of the generated pulse propagation regimes. The numerical model was solved with a standard symmetric split-step propagation algorithm, and the initial field was a picosecond Gaussian temporal profile.
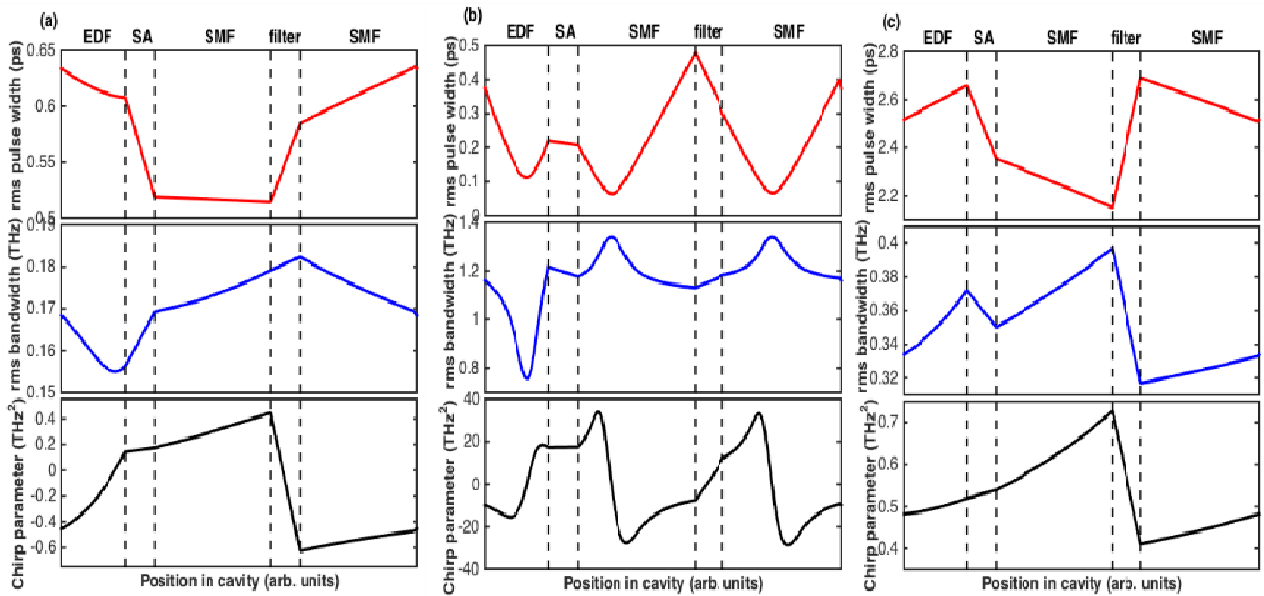

Figure 5. Simulated evolution of the rms temporal width, (red) rms spectral width (blue), and chirp parameter (black) of the pulse along the cavity for the (a) soliton, (b) DM soliton, and (c) DS regimes.

The corresponding simulated pulses and spectra (not shown) were in qualitative fairly good agreement with their experimental counterparts, with TBP values close to the experimental ones. The respective net GVD of the cavity was $0.397 \mathrm{ps}^{2}, 0 \mathrm{ps}^{2}$ and $0.502 \mathrm{ps}^{2}$, which are the same values as those of the experiments. The soliton-like pulse has FWHM temporal and spectral widths of $0.88 \mathrm{ps}$ and $2.85 \mathrm{~nm}$, yielding a TBP of 0.315 , which equals the transform limit for a hyperbolic secant pulse; the temporal and spectral widths of the DM soliton pulse are $2.65 \mathrm{ps}$ and $26 \mathrm{~nm}$, yielding a TBP of 8.61, and the DS features 3.51-ps pulse width and 10.24-nm bandwidth, thus a TBP of 4.49. These values are close to the experimental ones. Figure 5 shows the simulated evolution of the rms temporal width, (red) rms spectral width (blue), and chirp parameter (black) of the pulse along the cavity for the (a) soliton, (b) DM soliton, and (c) DS regimes. Distinctly different types of evolutions can be seen from this figure. The soliton-like pulse is nearly static in the cavity, with temporal and spectral breathing ratios (defined as the ratios of maximum and minimum rms widths within a roundtrip in the cavity) of only 1.3 and 1.2, respectively. The DM soliton experiences the largest temporal and spectral breathing, with the respective breathing ratios 7.5 and 1.8. This pulse temporally stretches and compresses three times per round-trip, reaches a minimum duration in the middle of each of EDF and two SMF segments, and acquires both signs of chirp. The mode-locked pulse at large net-normal dispersion exhibits the features of a DS with an evolution 
defined by the dispersion map [37]. The evolution in each fibre segment is monotonic, the pulse duration increases in the EDF segment and decreases in the SMF segments, and the spectrum, cut away by the filter, grows back in the fibre sections. The pulse is positively chirped throughout the cavity, and it depends strongly on dissipative effects such as the spectral filter and the SA. The different roles played by spectral filtering in the DM and DS mode-locking regimes are evident: in the DM soliton regime, the large filter has nearly no effect on the pulse spectrum, while the pulse is temporally compressed by the effect of the normal dispersion applied on the filter. In the DS regime, by contrast, the narrow filter cuts away the spectral structure and, consequently, the pulse duration is increased.

\section{CONCLUSIONS}

We have shown that dispersion management can be readily realized with an in-cavity spectral filter in passively modelocked fibre lasers. Soliton, DM soliton and DS soliton pulses can be selected and reliably targeted by only configuring the filter's dispersion and bandwidth. A numerical study has confirmed the proposed method. The peculiar properties of these nonlinear waves have been clearly revealed thanks to the special laser system in use. The manipulation of different pulse regimes in our laser is simple and reconfigurable, which is of great interest in the field of ultrafast optics. This multifunction ultrashort-pulse fibre laser is also attractive for optical communications, signal processing and other applications.

\section{ACKNOWLEDGEMENTS}

We would like to especially thank Prof. Christophe Finot for very helpful discussions and useful advice on how to improve this work. We also thank our colleagues Dr. Stylianos Sygletos and Dr. Marc Stephens, who provided us with the programmable filter. The research was supported by the European Commission under the Marie Curie International Incoming Fellowship scheme (grant no. 628198) and the Leverhulme Trust (UK, grant RPG-278).

\section{REFERENCES}

[1] U. Keller, "Recent developments in compact ultrafast lasers," Nature, 424(6950), 831-838 (2003).

[2] L. E. Nelson, D. J. Jones, K. Tamura et al., "Ultrashort-pulse fiber ring lasers," Applied Physics B-Lasers and Optics, 65(2), 277-294 (1997).

[3] I. N. Duling, "All-fiber ring soliton laser mode locked with a nonlinear mirror," Optics letters, 16(8), 539-541 (1991).

[4] D. Richardson, R. Laming, D. N. Payne et al., "320 fs soliton generation with passively mode-locked erbium fibre laser," Electronics Letters, 27(9), 730-732 (1991).

[5] K. Tamura, E. P. Ippen, H. A. Haus et al., "77-fs pulse generation from a stretched-pulse mode-locked all-fiber ring laser," Optics Letters, 18(13), 1080-1082 (1993).

[6] Y. Chen, F. Kärtner, U. Morgner et al., "Dispersion-managed mode locking," Journal of the Optical Society of America B, 16(11), 1999-2004 (1999).

[7] S. K. Turitsyn, B. G. Bale, and M. P. Fedoruk, "Dispersion-managed solitons in fibre systems and lasers," Physics reports, 521(4), 135-203 (2012).

[8] F. W. Wise, A. Chong, and W. H. Renninger, "High-energy femtosecond fiber lasers based on pulse propagation at normal dispersion," Laser \& Photonics Reviews, 2(1-2), 58-73 (2008).

[9] F. O. Ilday, J. R. Buckley, W. G. Clark et al., "Self-similar evolution of parabolic pulses in a laser," Physical Review Letters, 92(21), 213902/1-4 (2004).

[10] W. H. Renninger, A. Chong, and F. W. Wise, "Self-similar pulse evolution in an all-normal-dispersion laser," Physical Review A, 82(2), (2010).

[11] A. Chong, J. Buckley, W. Renninger et al., "All-normal-dispersion femtosecond fiber laser," Optics Express, 14(21), 10095-10100 (2006).

[12] J. Peng, "Gain dependent pulse regimes transitions in a dissipative dispersion-managed fibre laser," Optics Express, 24(3), 3046-3054 (2016).

[13] B. Oktem, C. Ulgudur, and F. O. Ilday, "Soliton-similariton fibre laser," Nature Photonics, 4(5), 307-311 (2010). 
[14] Z. Zhang, B. Öktem, and F. Ilday, "All-fiber-integrated soliton-similariton laser with in-line fiber filter," Optics letters, 37(17), 3489-3491 (2012).

[15] C. Aguergaray, D. Méchin, V. Kruglov et al., "Experimental realization of a mode-locked parabolic Raman fiber oscillator," Optics express, 18(8), 8680-8687 (2010).

[16] S. Boscolo, J. Fatome, S. K. Turitsyn Millot, G. \& Finot, C. "Temporal and spectral nonlinear pulse shaping methods in optical fibres," in All-optical signal processing: data communication and storage applications, Springer Series in Optical Science Vol. 94 (eds Wabnitz S. \& Eggleton, B. J.) 105-128 (Springer, 2015).

[17] B. Orta̧, M. Plötner, T. Schreiber et al., "Experimental and numerical study of pulse dynamics in positive netcavity dispersion modelocked Yb-doped fiber lasers," Optics express, 15(23), 15595-15602 (2007).

[18] M. Chernysheva, A. Krylov, A. Ogleznev et al., "Transform-limited pulse generation in normal cavity dispersion erbium doped single-walled carbon nanotubes mode-locked fiber ring laser," Optics express, 20(21), 23994-24001 (2012).

[19] N. Nishizawa, L. Jin, H. Kataura et al., "Dynamics of a Dispersion-Managed Passively Mode-Locked Er-Doped Fiber Laser Using Single Wall Carbon Nanotubes." 2, 808-824 (2015).

[20] R. Gumenyuk, I. Vartiainen, H. Tuovinen et al., "Dispersion compensation technologies for femtosecond fiber system," Applied optics, 50(6), 797-801 (2011).

[21] S. Boscolo, and S. K. Turitsyn, "Intermediate asymptotics in nonlinear optical systems," Physical review A, 85(4), 043811 (2012).

[22] J. Peng, L. Zhan, Z. Gu et al., "Experimental observation of transitions of different pulse solutions of the Ginzburg-Landau equation in a mode-locked fiber laser," Physical Review A, 86(3), 033808 (2012).

[23] A. M. Weiner, "Femtosecond pulse shaping using spatial light modulators," Review of scientific instruments, 71(5), 1929-1960 (2000).

[24] J. Schröder, T. D. Vo, and B. J. Eggleton, "Repetition-rate-selective, wavelength-tunable mode-locked laser at up to $640 \mathrm{GHz}$," Optics letters, 34(24), 3902-3904 (2009).

[25] X. Yang, K. Hammani, D. J. Richardson et al., "Passively mode-locked fiber laser incorporating adaptive filtering and dispersion management," In Conference on Lasers and Electro-Optics, OSA Technical Digest, paper CM1I.1(Optical Society of America, 2013). (2013).

[26] J. B. Schroeder, S. Coen, T. Sylvestre et al., "Dark and bright pulse passive mode-locked laser with in-cavity pulse-shaper," Optics express, 18(22), 22715-22721 (2010).

[27] S. Boscolo, C. Finot, H. Karakuzu et al., "Pulse shaping in mode-locked fiber lasers by in-cavity spectral filter," Optics letters, 39(3), 438-441 (2014).

[28] S. Boscolo, C. Finot, and S. K. Turitsyn, "Bandwidth Programmable Optical Nyquist Pulse Generation in Passively Mode-Locked Fiber Laser," Photonics Journal, IEEE, 7(5), 1-9 (2015).

[29] K. Tamura, H. A. Haus, and E. P. Ippen, "Self-starting additive pulse mode-locked Erbium fiber ring laser," Electronics Letters, 28(24), 2226-2228 (1992).

[30] H. Haus, E. Ippen, and K. Tamura, "Additive-pulse modelocking in fiber lasers," Quantum Electronics, IEEE Journal of, 30(1), 200-208 (1994).

[31] S. Kelly, "Characteristic sideband instability of periodically amplified average soliton," Electronics Letters, 28(8), 806-807 (1992).

[32] A. Chong, W. H. Renninger, and F. W. Wise, "All-normal-dispersion femtosecond fiber laser with pulse energy above 20 nJ," Optics Letters, 32(16), 2408-2410 (2007).

[33] A. Cabasse, B. Ortac, G. Martel et al., "Dissipative solitons in a passively mode-locked Er-doped fiber with strong normal dispersion," Optics Express, 16(23), 19322-19329 (2008).

[34] J. Peng, L. Zhan, Z. Gu et al., "Direct generation of 4.6-nJ 78.9-fs dissipative solitons in an all-fiber net-normaldispersion Er-doped laser," Photonics Technology Letters, IEEE, 24(2), 98-100 (2012).

[35] A. Chong, W. H. Renninger, and F. W. Wise, "Properties of normal-dispersion femtosecond fiber lasers," Journal of the Optical Society of America B, 25(2), 140-148 (2008).

[36] H. A. Haus, "Mode-locking of lasers," IEEE Journal of Selected Topics in Quantum Electronics, 6(6), 11731185 (2000).

[37] B. G. Bale, S. Boscolo, and S. K. Turitsyn, "Dissipative dispersion-managed solitons in mode-locked lasers," Optics Letters, 34(21), 3286-3288 (2009). 